Copyright by the American Physical Society. Feng, G. F.; Zallen, R., "Optical properties of ion-implanted GaAs: The observation of finite-size effects in GaAs microcrystals, "Phys. Rev. B 40, 1064 DOI: http://dx.doi.org/10.1103/

\title{
Optical properties of ion-implanted GaAs: The observation of finite-size effects in GaAs microcrystals
}

\author{
G. F. Feng and R. Zallen \\ Department of Physics, Virginia Tech, Blacksburg, Virginia 24061
}

(Received 17 October 1988; revised manuscript received 16 March 1989)

\begin{abstract}
We have carried out reflectivity measurements, for photon energies from 2.0 to $5.6 \mathrm{eV}$ in the electronic interband regime, for a series of unannealed ion-implanted GaAs samples which had been exposed to $45-\mathrm{keV} \mathrm{Be}^{+}$ions at various fluences up to $5 \times 10^{14} \mathrm{ions} / \mathrm{cm}^{2}$. The microstructure of the near-surface implantation-induced damage layer in these samples is known (from previous Raman work) to consist of a fine-grain mixture of amorphous GaAs and GaAs microcrystals, with the characteristic microcrystal size $L$ of the microcrystalline component decreasing with increasing fluence $\left(L=55 \AA\right.$ at $\left.5 \times 10^{14} \mathrm{~cm}^{-2}\right)$. The optical dielectric function of each sample's damage layer has been derived from the observed reflectivity spectrum by Lorentz-oscillator analysis. Then, by inverting the effective-medium approximation, we have extracted the dielectric function of the microcrystalline component ( $\mu$-GaAs) within the damage layer. The optical properties of $\mu$-GaAs differ appreciably from those of the bulk crystal, the difference increasing with decreasing $L$. We find that the microcrystallinity-induced spectral changes are concentrated in the linewidths of the prominent interband features $E_{1}, E_{1}+\Delta_{1}$, and $E_{2}$. These linewidths increase linearly and rapidly with inverse microcrystal size: $\Gamma_{\mu}=\Gamma_{0}+A L^{-1}$, where $\Gamma_{0}$ is the linewidth in the bulk crystal, $\Gamma_{\mu}$ is the linewidth in $\mu$-GaAs, and $A$ is a constant. For the $E_{1}$ and $E_{2}$ peaks, the experimentally determined value of $A$ is such that the finite-size broadening $\left(A L^{-1}\right)$ is about $0.2 \mathrm{eV}$ when $L=100 \AA$. We propose a simple theory of the finite-size effects which, when combined with band-structure information for GaAs, semiquantitatively accounts for our observations. Small microcrystal size implies a short time for an excited carrier to reach, and be scattered by, the microcrystal boundary, thus limiting the excited-state lifetime and broadening the excited-state energy. An alternative uncertainty-principle argument is also given in terms of the confinement-induced $\mathbf{k}$-space broadening of electron states.
\end{abstract}

\section{INTRODUCTION}

Ion-processed semiconductors are of both technological importance and scientific interest. Structural damage is inevitable in processes such as ion implantation ${ }^{1,2}$ and ion etching. ${ }^{3,4}$ Near-surface damage in semiconductors produced by light-ion species, such as $\mathbf{B}^{+}$and $\mathrm{Be}^{+}$, has been studied with various experimental techniques such as ellipsometry, ${ }^{1}$ Raman scattering, ${ }^{2,5}$ and transmission electron microscopy. ${ }^{6}$ Unlike heavy-ion implantation, light-ion implantation at room temperature with moderate energy and fluence (or dose) does not produce a layer made completely amorphous. ${ }^{6-8}$

In a recent investigation of the near-surface damage layer in unannealed $\mathrm{Be}^{+}$-implanted GaAs, Holtz et al. ${ }^{2}$ have used a combination of Raman scattering and chemical etch to obtain microstructural information for samples exposed to various fluences. The damage layer was shown to consist of amorphous GaAs (a-GaAs) and GaAs microcrystals ( $\mu$-GaAs), and they derived (for each implant) the microcrystalline volume fraction, the characteristic microcrystal size, and the depth profile. In the present paper we report the results of a visible and ultraviolet investigation of the electronic interband spectra for these same Raman-characterized $\mathrm{Be}^{+}$-implanted $\mathrm{GaAs}$ samples. One purpose of these experiments is to determine the effect of microcrystal size in the $100-\AA$ range on the optical properties and electronic excitations of microcrystalline GaAs. Microcrystalline Si has recently been extensively investigated, ${ }^{9-11}$ but little is known about microcrystalline GaAs. The microstructural information available for the $\mathrm{Be}^{+}$-implanted material provides us with an opportunity to correlate our results on $\mu$ GaAs with microcrystal size, and to make quantitative statements about finite-size effects in $\mu$-GaAs.

Polycrystalline semiconductors with crystallite sizes in the range below $500 \AA$ (say 50-500 $\AA$, or 5-50 nm) are usually termed microcrystalline, ${ }^{10}$ although nanocrystalline is also sometimes used. ${ }^{11}$ Throughout this paper we use the term microcrystalline GaAs (symbolized here by $\mu$-GaAs) to denote the crystalline component in our implanted GaAs samples, which we know (from the Raman work $^{2}$ ) to be characterized by microcrystal sizes in this range.

We present results of visible-ultraviolet reflectivity measurements which probe the nature of electronic excitations within the damage layer of $\mathrm{Be}^{+} \rightarrow \mathrm{GaAs}$. The experimental methods and observed reflectivity spectra are described in Sec. II. In Sec. III the optical dielectric function of the damage layer of each implanted material is derived by means of Lorentz-oscillator analysis. The optical properties of $\mu-\mathrm{GaAs}$, the microcrystalline com- 
ponent, is extracted in Sec. IV with the use of the effective-medium approximation (EMA). Section $V$ presents our main findings on finite-size electronic effects in $\mu$-GaAs. The microcrystallinity-induced spectral changes are concentrated in the linewidths of the three sharp interband peaks; these linewidths increase linearly and rapidly with increasing inverse microcrystal size. A simple theory, discussed in Sec. VI, semiquantitatively accounts for our observations. Small microcrystal size implies a short time for an excited carrier to reach, and to be scattered by, the microcrystal boundary, thus limiting the excited-state lifetime and broadening the excitedstate energy. An alternative uncertainty-principle argument can be given in terms of confinement-induced $k$ space broadening of electron states. A summary of the paper is given in Sec. VII.

\section{EXPERIMENT}

The c-GaAs starting material was Czochralski-grown, chromium-doped, semi-insulating, single-crystal GaAs. (100)-oriented wafers were cut, polished, then etched in 8:1:1 $\mathrm{H}_{2} \mathrm{SO}_{4}: 30 \mathrm{~mol} \% \mathrm{H}_{2} \mathrm{O}_{2}: \mathrm{H}_{2} \mathrm{O}$ prior to implanting at room temperature. Implantation was carried out at the Texas Instruments Central Research Laboratory, using $45-\mathrm{keV} \mathrm{Be}^{+}$ions incident at $9^{\circ}$ from the normal to avoid channeling. Ion fluences ranged from $1 \times 10^{13}$ to $5 \times 10^{14}$ ions $/ \mathrm{cm}^{2}$. No anneal was done following implantation. The samples were of excellent optical quality; no implantation-induced surface roughness was discernible with laser-beam scattering.

Reflectivity measurements in the visible and nearultraviolet region (photon energy $E$ in the range 2-5.6 eV) were performed with a modified Perkin-Elmer prism-grating spectrometer, using chopped radiation and lock-in detection. The spectrometer was controlled with a NEC APCIII personal computer, which also performed data acquisition from the lock-in amplifier. Reflectivity was calibrated using the reflectivity of $c$-GaAs derived from ellipsometry measurements ${ }^{12}$ as a reference standard. Our samples were exposed to air, and the thin oxide layer present on air-exposed GaAs is known to modify the observed reflectivity. ${ }^{12,13}$ In order to correct for this effect (a reflectivity reduction occurring mainly at photon energies above $4.5 \mathrm{eV}$ ), we used, as the reference standard for our $c$-GaAs sample, the reflectivity spectrum of oxide-free $c$-GaAs reported by Aspnes and Studna. ${ }^{12}$ This provided a spectral correction for use with the aluminum mirror against which all our samples were compared. The same correction (which has the effect of "optically removing" the oxide layer for our c-GaAs sample) was then applied to our measurements on the ionimplanted samples. For these, the correction is expected to be only approximate. We estimate the error in reflectivity to be at worst $3 \%$ for photon energies above 5 $\mathrm{eV}$, and less at lower photon energies (reflectivity reproducibility was within $1 \%$ ). Our results of Sec. V may be slightly affected by this, but the effect on the lower energy $E_{1}$ and $E_{1}+\Delta_{1}$ peaks should be negligible. Photon energy was known within $0.01 \mathrm{eV}$ throughout the spectral re- gion covered. All measurements were carried out at room temperature.

Figure 1 displays our results for the reflectivity spectra $\boldsymbol{R}(\boldsymbol{E})$ of four beryllium-implanted GaAs samples. Panels (a), (b), (c), and (d) show the spectra observed after implantation $\left(45-\mathrm{keV} \mathrm{Be}^{+}\right.$ions) with fluences of $1 \times 10^{13}$, $5 \times 10^{13}, 1 \times 10^{14}$, and $5 \times 10^{14}$ ions $/ \mathrm{cm}^{2}$, respectively. In addition to the experimental data on the ion-implanted samples, indicated by the points, each panel also contains two curves which correspond to the spectra of crystalline GaAs (labeled $c$ ) and of amorphous GaAs (labeled $a$ ). These two curves are repeated in each panel in order to provide visual benchmarks for the evolution of the GaAs spectrum with increasing implantation dose. The amorphous spectrum shown corresponds to implantationamorphized $\mathrm{GaAs}$ produced by high-fluence high-energy $\mathrm{As}^{+}$bombardment. ${ }^{14,15}$ A systematic spectral change is clear in Fig. 1; the higher the ion fluence, the more severe the deviation from the spectrum of the bulk crystal.

\section{THE DIELECTRIC FUNCTION OF THE IMPLANTED MATERIAL}

In order to derive the complex dielectric function and the other optical properties from each measured

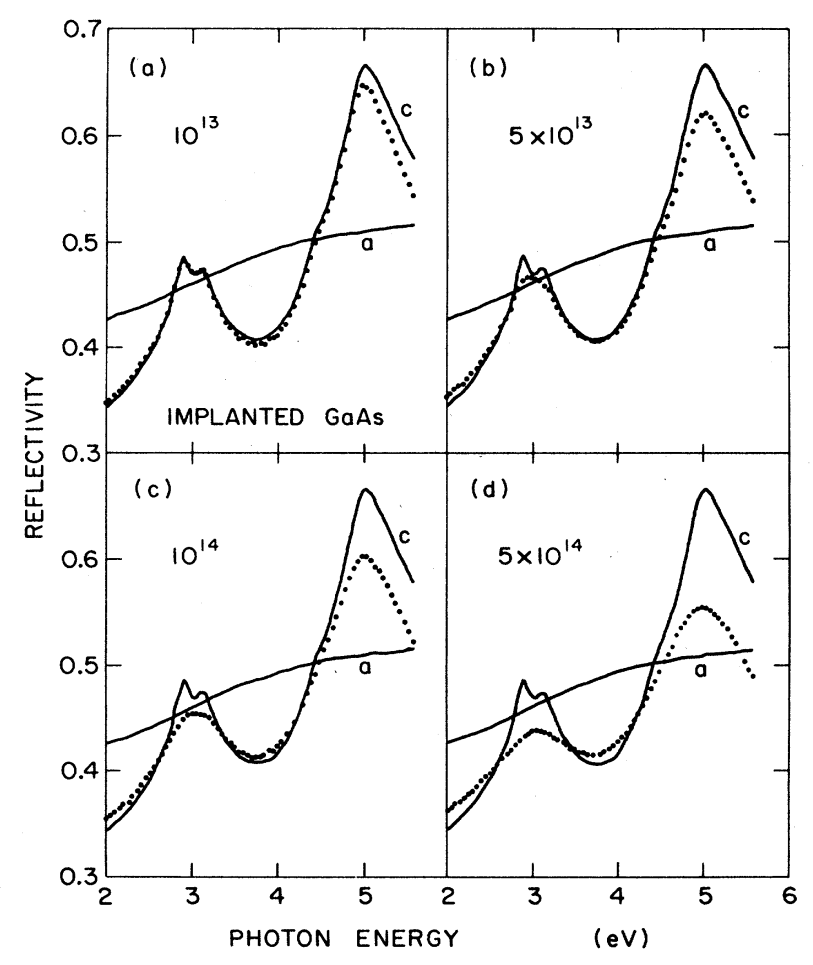

FIG. 1. Reflectivity spectra, shown as points, of $45-\mathrm{keV}$ $\mathrm{Be}^{+}$-implanted GaAs with ion fluence (a) $1 \times 10^{13}$, (b) $5 \times 10^{13}$, (c) $1 \times 10^{14}$, and (d) $5 \times 10^{14}$ ions $/ \mathrm{cm}^{2}$. The two curves included for comparison, and repeated in each panel, are the reflectivity spectra of $c$-GaAs and $a$-GaAs. $R$ is plotted against the photon energy $E=\hbar \omega, \omega$ being the angular frequency. 
reflectivity spectrum, a Lorentz-oscillator expression ${ }^{1,16,17}$ was used for the dielectric function:

$$
\epsilon(\omega)=\epsilon_{\infty}+\sum_{i=1}^{n} \frac{F_{i}^{2}}{\left(\omega_{i}^{2}-\omega^{2}\right)-i \Gamma_{i} \omega} .
$$

Here, $\epsilon(\omega)$ is the dielectric function at frequency $\omega, n$ is the number of oscillators, $F_{i}, \omega_{i}$, and $\Gamma_{i}$ are the strength, position, and linewidth parameters of the $i$ th oscillator, and $\epsilon_{\infty}$ is one additional parameter corresponding to the high-frequency asymptote. Reflectivity can be determined directly from the real $\left(\epsilon_{1}\right)$ and imaginary $\left(\epsilon_{2}\right)$ parts of $\epsilon$ : $\epsilon=\epsilon_{1}+i \epsilon_{2}$. In this way a calculated reflectivity spectrum $R(\omega)$ can be generated from the set of $3 n+1$ parameters, $F_{i}, \omega_{i}, \Gamma_{i}$, and $\epsilon_{\infty}$. The calculated reflectivity spectrum is then compared with the experimental one. A computer program was developed, based on nonlinear least-squares fitting, ${ }^{18}$ to obtain the parameter set that gives the best fit of the calculated to the experimental reflectivity spectrum.

An error criterion $\delta$, corresponding to a normalized average square deviation, was defined as

$$
\delta=\frac{\sum_{i=1}^{N}\left|f_{\mathrm{fit}}\left(\omega_{i}\right)-f_{\exp }\left(\omega_{i}\right)\right|^{2}}{\sum_{i}\left|f_{\exp }\left(\omega_{i}\right)\right|^{2}}
$$

where $N$ is the number of data points, and $f$ is the data function, which can be the reflectivity, the dielectric function, or other functions.

We found that seven oscillators were needed to adequately fit the reflectivity spectrum of each sample. Seven oscillators were also used in the ellipsometry studies of Erman et al. ${ }^{1}$ The fitting technique was checked by applying it to $c$-GaAs, for which the reflectivity, the dielectric function, and the other related optical properties are rather accurately known from ellipsometry measurements. ${ }^{12}$ Oscillator fitting was carried out on the ellipsometry-derived reflectivity function $R(E)$. The error criterion $\delta$ [Eq. (2), with $f=R$ ] was less than $10^{-4}$. Also, the dielectric function $\epsilon(\omega)$ corresponding [via Eq. (1)] to the fitted set of oscillator parameters was compared with the $\epsilon(\omega)$ values measured ellipsometrically. ${ }^{12}$ The corresponding error $\delta$ [Eq. (2), with $f=\epsilon$ ] was about $10^{-3}$. In addition, a different fit was also performed in which the ellipsometry-derived dielectric function, rather than the reflectivity, was the fitted quantity. This yielded a $\delta$ of about $10^{-4}$. All of these tests show that the fitting procedure is satisfactory. We did find that the error is not uniformly distributed; deviations tend to be larger in regions in which either $\epsilon_{1}$ or $\epsilon_{2}$ is small. In these regions, ellipsometry measurements also involve a large uncertainty. ${ }^{2}$

The dielectric functions of the implanted samples and of $c$-GaAs were thus obtained by oscillator fits of the measured reflectivity, using seven oscillators for each sample. In all of the fits the value of the error criterion $\delta$ $(f=R)$ was less than $10^{-4}$. The real part, $\epsilon_{1}(E)$, and the imaginary part, $\epsilon_{2}(E)$, of the dielectric functions of the implanted samples are plotted in Figs. 2 and 3, respec-

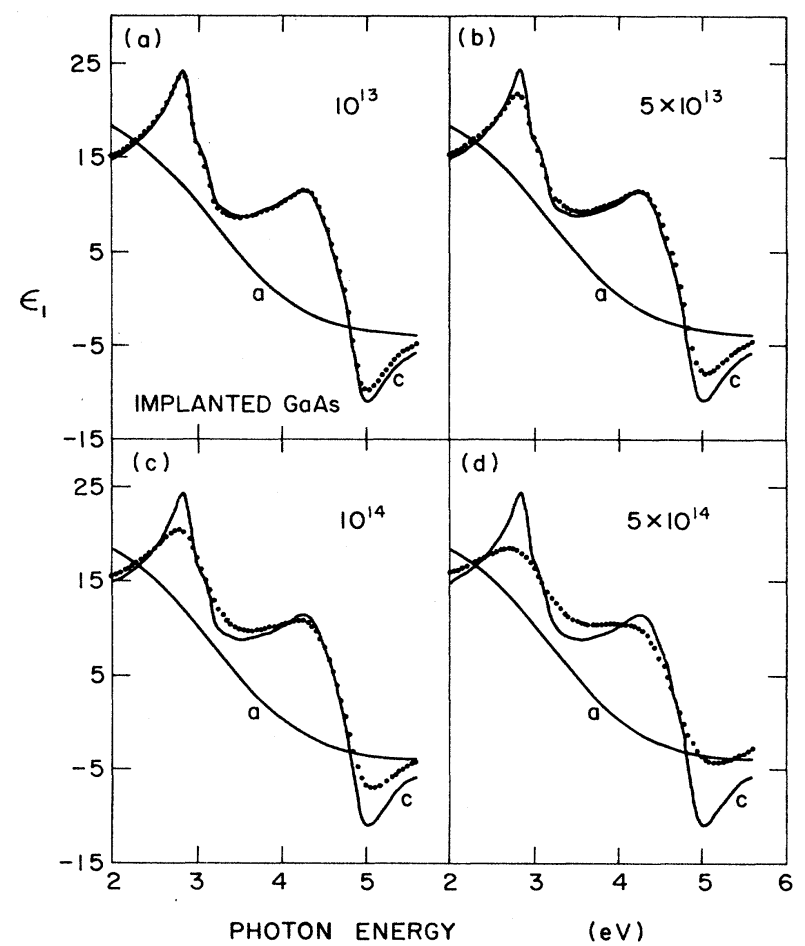

FIG. 2. Real part of the dielectric function of $45-\mathrm{keV} \mathrm{Be}^{+}$. implanted GaAs with ion fluence (a) $1 \times 10^{13}$, (b) $5 \times 10^{13}$, (c) $1 \times 10^{14}$, and (d) $5 \times 10^{14}$ ions $/ \mathrm{cm}^{2}$. The dielectric functions are obtained from the reflectivity spectra of Fig. 1 using Lorentzoscillator analysis, as described in the text. The $c$-GaAs and $a$ GaAs counterparts are included for comparison.

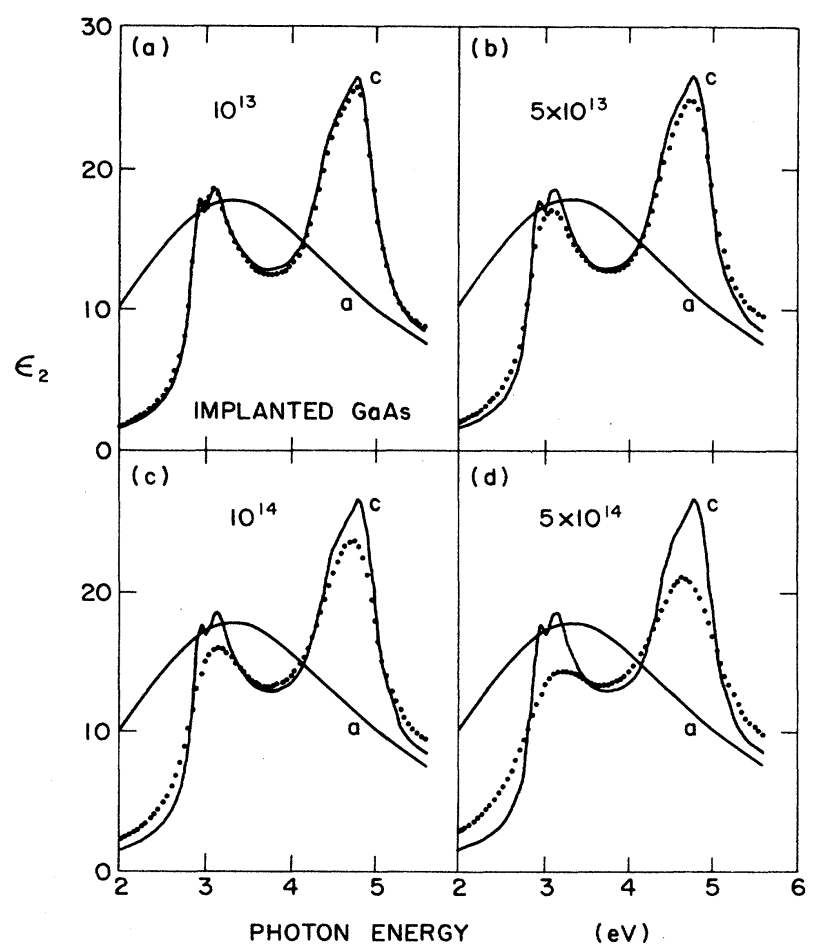

FIG. 3. Imaginary part of the dielectric functions of the implanted samples, along with the $c$-GaAs and $a$-GaAs counterparts. 
tively, along with their crystal and amorphous counterparts. Again, the $a$-GaAs spectra are from the work of Aspnes on implantation-amorphized GaAs. ${ }^{14}$ The dielectric function is seen to be sensitive to the damage produced by ion implantation. The sharp spectral features are smeared out as ion fluence increases. These spectral changes are due to two quite distinct effects: the increasing presence of the amorphous phase and the finite-size effects on the optical properties of the crystalline phase. The first is self-explanatory. We shall concentrate our attention on the second effect.

\section{OPTICAL PROPERTIES OF THE MICROCRYSTALLINE COMPONENT}

The structure of ion-implanted semiconductors is believed to have the nature of a volume mixture of microcrystals and amorphous material. ${ }^{19}$ In particular, the recent Raman studies on the same samples studied here strongly support a structural model of the implantationinduced damage layer as a fine-scale mixture of amorphous and microcrystalline GaAs. ${ }^{2}$ Also, these samples $\left(45-\mathrm{keV} \mathrm{Be}^{+}\right.$implants to fluences up to $5 \times 10^{14} \mathrm{~cm}^{-2}$ ) were shown, by chemical-etch depth-profile Raman studies, ${ }^{2}$ to have a uniform high-damage plateau extending from the surface to a depth of about $1500 \AA$, followed by a graded transition region in which the structure varies with depth until the undamaged crystal substrate is reached at about $4000 \AA$. We have made reflectivity measurements on these samples following chemical-etch removal of near-surface layers, and find that the uv spectrum exhibits a similar depth profile.

In our reflectivity work, photon energies exceeded $2 \mathrm{eV}$ (well above the room-temperature band gap of $1.43 \mathrm{eV}$ ) and the optical penetration depth for this strongly absorbed light was less than the depth of the high-damage plateau. This means that we probed a region with macroscopically uniform structure, avoiding the complication of the graded transition region. Another significant feature of these samples, as will be seen in the next section, is that the microcrystalline component dominates the damage layer.

We use the Bruggeman effective-medium approximation $^{20,21}$ (EMA), a self-consistent effective-medium theory, to analyze the optical properties of our system. When applied to our two-phase medium, the EMA takes the form

$$
\frac{\epsilon-\epsilon_{a}}{2 \epsilon+\epsilon_{a}} f_{a}+\frac{\epsilon-\epsilon_{\mu}}{2 \epsilon+\epsilon_{\mu}} f_{\mu}=0 .
$$

Here, $\epsilon, \epsilon_{\mu}$, and $\epsilon_{a}$ denote the complex dielectric functions of the implanted material, the microcrystalline phase, and the amorphous phase; $f_{a}$ and $f_{\mu}\left(f_{\mu}=1-f_{a}\right)$ are the volume fractions of the amorphous and microcrystalline phases, respectively.

The dielectric function $\epsilon_{a}$ of $a$-GaAs is broad and featureless in this spectral region, and we assume that the amorphous component in the implanted material has the same dielectric function. But we do not assume that the dielectric function of the microcrystalline component, $\epsilon_{\mu}$, is the same as that of $c$-GaAs, i.e., we do not assume that $\epsilon_{\mu}=\epsilon_{c}$. The sharp spectral features of $\epsilon_{c}$ are due to interband $\mathbf{k}$-conserving transitions at critical points in $\mathbf{k}$ space, and would be expected to be sensitive to finite-size effects in small microcrystals. Our analysis is thus focused on the optical properties of $\mu$-GaAs, the microcrystalline component within the damage layer of our implanted GaAs samples. We therefore use the EMA to derive $\epsilon_{\mu}$ from the measured $\epsilon$ and the known $\epsilon_{a}$.

The dielectric function of the microcrystalline phase is obtained by solving Eq. (3) for $\epsilon_{\mu}$ :

$$
\epsilon_{\mu}=\frac{1+2 B}{1-B} \epsilon
$$

where

$$
B \equiv \frac{\epsilon-\epsilon_{a}}{2 \epsilon+\epsilon_{a}} \frac{f_{a}}{1-f_{a}} .
$$

In order to obtain $f_{a}$, the amorphous volume fraction, it is assumed that away from the sharp interband features of the bulk dielectric function, at $2.9 \mathrm{eV}\left(E_{1}\right), 3.1 \mathrm{eV}$ $\left(E_{1}+\Delta_{1}\right)$, and $4.9 \mathrm{eV}\left(E_{2}\right)$, the optical properties of the microcrystallites are close to those of the bulk crystal $\left(\epsilon_{\mu}=\epsilon_{c}\right)$. This assumption is supported by the good agreement of the $f_{a}$ values obtained by this procedure with those obtained independently for these samples from the earlier Raman studies. ${ }^{2}$ It is also self-consistent, as shown later in the $\alpha_{\mu}$ spectra of Fig. 6, where $\alpha_{\mu}$ is the microcrystalline-phase optical-absorption coefficient. The amorphous volume fraction of each sample can thus be estimated by using the following simple relation, ${ }^{2}$ valid only in spectral regions away from the critical-point features of $\epsilon_{c}$, connecting the absorption coefficients of the bulk crystal $\left(\alpha_{c}\right)$, the amorphous phase $\left(\alpha_{a}\right)$, and the implanted material $(\alpha)$ :

$$
\alpha=\alpha_{a} f_{a}+\alpha_{c}\left(1-f_{a}\right) .
$$

The spectral region below $2.5 \mathrm{eV}$, well below the first strong interband feature at $2.9 \mathrm{eV}$, was chosen to calculate $f_{a}$. In Eq. (6), $\alpha$ and $\alpha_{c}$ are known from our experiments (determined from the dielectric functions of Figs. 2 and 3), while $\alpha_{a}$ is known from previous work. ${ }^{14}$ Obviously, $\alpha_{a}$ and $\alpha_{c}$ need to be appreciably different from each other, and $\alpha$ must be intermediate between them, in order for Eq. (6) to be useful for estimating $f_{a}$. These conditions are indeed met in the stated special region, in which $\alpha_{a}$ is a factor of 4-5 larger than $\alpha_{c}$.

The amorphous fractions estimated from Eq. (6) are listed in Table I, along with the Raman-derived results ${ }^{2}$ obtained previously. The last column in the table is the characteristic microcrystallite size $L$ deduced from the Raman measurements by analyzing the size-effectinduced shifting and broadening of the longitudinaloptical-phonon line. ${ }^{2}$ The amorphous fraction is relatively low, which is consistent with the idea that relatively light damage is induced by light-ion implantation at room temperature. For the most heavily damaged sample, the crystalline component still accounts for 75-80\% of the volume fraction. But the characteristic microcrys- 
TABLE I. Amorphous fraction and crystallite size in $\mathrm{Be}^{+} \rightarrow \mathrm{GaAs}$.

\begin{tabular}{ccccc}
\hline \hline Sample & Fluence $\left(\mathrm{cm}^{-2}\right)$ & $f_{a}(\text { opt })^{\mathrm{a}}$ & $f_{a}(\text { Raman })^{\mathrm{b}}$ & $L(\text { Raman })^{\mathrm{b}}(\AA)$ \\
\hline (a) & $1 \times 10^{13}$ & 0.03 & & $(500)^{\mathrm{c}}$ \\
(b) & $5 \times 10^{13}$ & 0.07 & 0.07 & 160 \\
(c) & $1 \times 10^{14}$ & 0.11 & 0.12 & 82 \\
(d) & $5 \times 10^{14}$ & 0.20 & 0.25 & 55 \\
\hline \hline
\end{tabular}

${ }^{a}$ Estimated from the optical studies reported here.

${ }^{b}$ Estimated from the Raman studies of Ref. 2.

${ }^{c}$ Crude estimate based on Fig. 7. The other entries in the last column are based on Ref. 2.

tallite size $L$ is very small; $L \approx 55 \AA$ for the $5 \times 10^{14}-\mathrm{cm}^{2}$ implant.

The dielectric functions of the microcrystalline phase of the implanted samples, calculated using Eqs. (4) and (5) and the $f_{a}$ values in Table I (column 2), are presented in Figs. 4 and 5. These spectra clearly show that the optical properties of $\mu$-GaAs are substantially different than those of $c$-GaAs, especially in the vicinity of the sharp interband features. The $E_{1}$ and $E_{1}+\Delta_{1}$ doublet of $\epsilon_{2}$ merges into a single peak as crystallite size becomes less than about $200 \AA$.

The optical-absorption spectrum of the microcrystalline component of each sample, denoted $\alpha_{\mu}(E)$, was

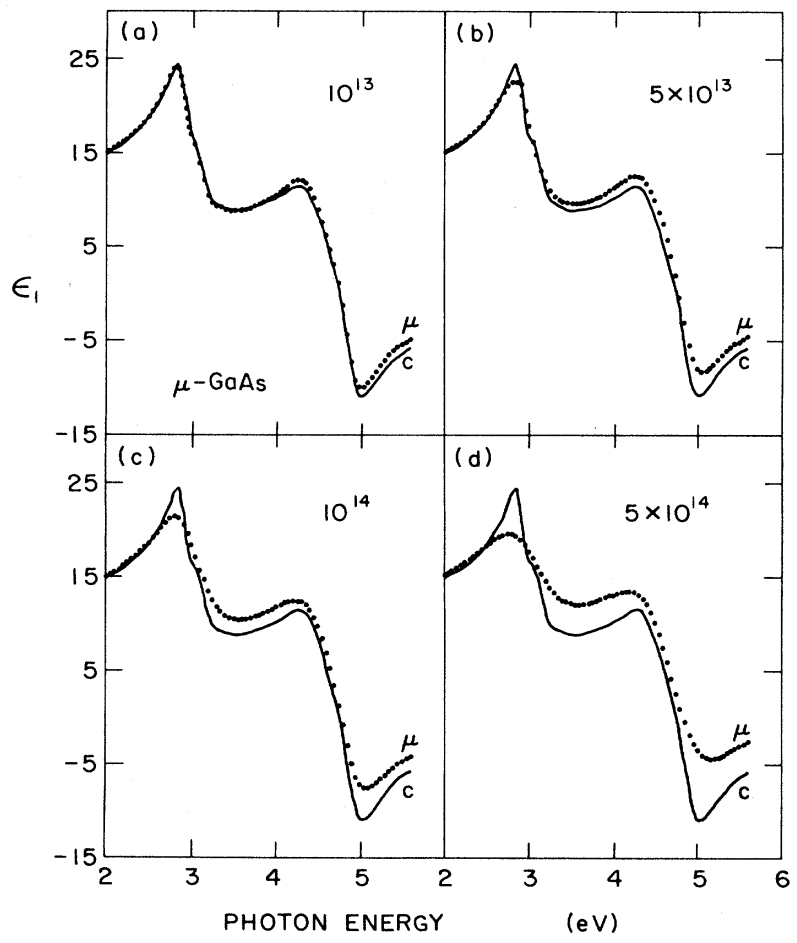

FIG. 4. Real part of the dielectric function of the microcrystalline phase ( $\mu$-GaAs) in $45-\mathrm{keV} \mathrm{Be}^{+}$-implanted $\mathrm{GaAs}$ with ion fluence (a) $1 \times 10^{13}$, (b) $5 \times 10^{13}$, (c) $1 \times 10^{14}$, and (d) $5 \times 10^{14}$ ions $/ \mathrm{cm}^{2}$. The dielectric functions of $\mu$-GaAs are deduced from the results of Figs. 2 and 3 using the effective-medium approximation, as described in the text. The $c$-GaAs counterpart is included in each panel for comparison. determined in each case from the microcrystalline-phase dielectric function $\epsilon_{\mu}(E)$, whose real and imaginary parts were shown in Figs. 4 and 5. The results for $\alpha_{\mu}(E)$ are shown in Fig. 6, where they are compared with the bulkcrystal spectrum $\alpha_{c}(E)$. We see that below $2.5 \mathrm{eV} \alpha_{\mu}$ is close to $\alpha_{c}$, which is self-consistent with (and thereby supports) the assumption made above in the calculation of $f_{a}$.

However, throughout much of the spectral range displayed in Figs. 4-6 the optical properties of $\mu$-GaAs are seen to differ appreciably from the optical properties of $c$-GaAs. This is a key result.

The dielectric function of $\mu$-GaAs is different for each implant, i.e., $\epsilon_{\mu}$ changes with ion fluence. The physical reason for this is that $\epsilon_{\mu}$ depends on the characteristic microcrystallite size $L$ within the microcrystalline phase. $L$ is an internal variable which controls the properties of $\mu$ -

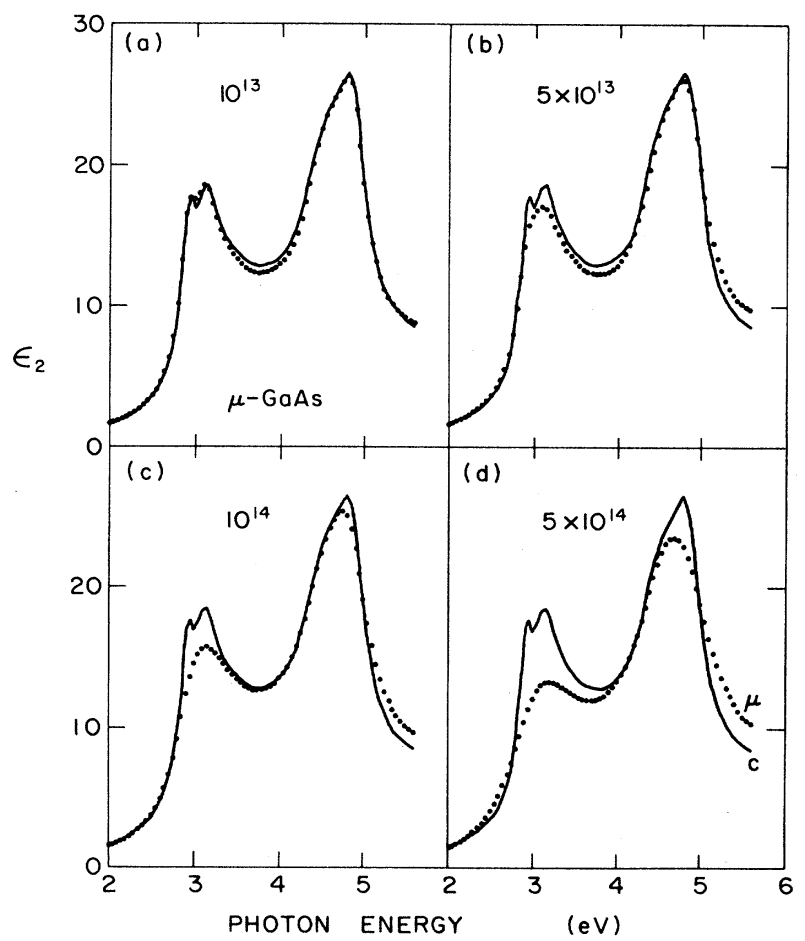

FIG. 5. Imaginary part of the dielectric function of the microcrystalline phase in each of the implanted samples, as described in the caption of Fig. 4. 


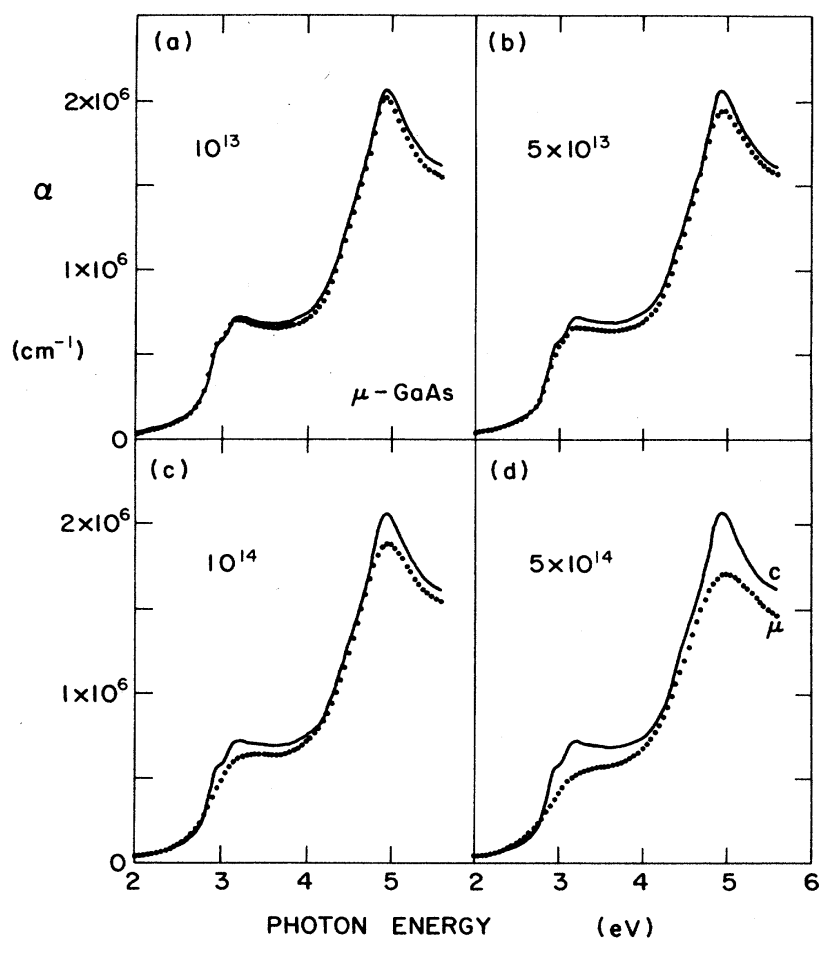

FIG. 6. Absorption coefficient of the microcrystalline phase in each of the implanted samples, as described in the caption of Fig. 4. The absorption coefficients are directly calculated from the dielectric functions of Figs. 4 and 5.

GaAs. We know $L$ from the Raman work, and we know that it changes from sample to sample (Table I). Our experiments thus yield the $L$ dependence of $\epsilon_{\mu}$, which becomes progressively more different from the bulk-crystal $\epsilon_{c}$ as $L$ decreases. The observed $L$ dependence is analyzed in Sec. V and interpreted in Sec. VI.

\section{THE EFFECTS OF MICROCRYSTAL SIZE ON THE OPTICAL PROPERTIES OF $\mu$-GaAs}

Figures 4-6 display our results for the optical properties of $\mu$-GaAs, the microcrystalline phase that is the main component within the near-surface damage layer of the implanted GaAs samples. A clear evolution is seen with decreasing microcrystal size. To quantitatively characterize this evolution, a second oscillator analysis was now performed on the microcrystalline-phase dielectric function $\epsilon_{\mu}$, for each of the four $\mu$-GaAs phases studied. Since oscillator parameters characterize the $\epsilon_{\mu}$ spectra in a consistent way, systematic microcrystallinityinduced changes should be significant.

In Table II we compare the oscillator parameters found for $c$-GaAs with those found for the $\mu$-GaAs phase in the $5 \times 10^{14}-\mathrm{cm}^{-2}$ implant. This corresponds to the comparison between the $c$-GaAs and $\mu$-GaAs spectra shown in panel (d) of Figs. 4-6. For this $\mu$-GaAs the characteristic microcrystal size is about $55 \AA$ (Table I).

A quick review of the road to Table II is in order. Oscillator analysis of the measured reflectivity (Fig. 1) was used to determine the dielectric function (Figs. 2 and 3) of the implanted material. Effective-medium analysis [Eqs. (4) and (5)] was then used to extract the dielectric function $\epsilon_{\mu}$ of the $\mu$-GaAs component, using the composition $\left(f_{\mu}, f_{a}\right)$ and dielectric function of the implanted material and the known dielectric function $\epsilon_{a}$ of $a-\mathrm{GaAs}$. Finally, in order to track the changes that occur with decreasing $L$, oscillator parameters were fitted to the $\epsilon_{\mu}$ spectra of Figs. 4 and 5.

Among the oscillators listed in Table II, three have especially small (less than $0.4 \mathrm{eV}$ ) $c$-GaAs linewidth values $\Gamma_{i}(c)$. These three oscillators correspond closely to the three prominent sharp features in the crystalline spectrum. They are identified with the direct interband transitions, $E_{1}, E_{1}+\Delta_{1}$, and $E_{2}$, and their oscillator energies, $2.92,3.12$, and $4.85 \mathrm{eV}$, are close to the accepted room-temperature transition energies. ${ }^{13}$ We shall focus our attention on these three labeled rows in Table II. Microcrystallinity scarcely affects these transition energies; $E_{i}(\mu)$ differs from $E_{i}(c)$ by no more than $0.04 \mathrm{eV}$ for these three oscillators, and we do not view these differences as experimentally significant. Skipping over to the last two columns, we see definite $c \rightarrow \mu$ changes (10-15\%, both signs) in the fitted $F_{i}$ values. However, these $F_{i}$ changes, like the $E_{i}$ changes, are overshadowed by the striking changes manifest in the middle two columns of the table, and we proceed immediately to dis-

TABLE II. Comparison of the oscillator parameters [Eq. (1)] fitted to $c$-GaAs and to the microcrystalline phase $\left(\mu\right.$-GaAs) in the $5 \times 10^{14}-\mathrm{cm}^{-2}$ implant. (The parameter values listed in the table have been rounded off from the actual fitting parameters; the dropped digits are not significant and contribute only noise to a discussion of these values.)

\begin{tabular}{|c|c|c|c|c|c|c|}
\hline $\begin{array}{l}\text { Interband } \\
\text { transition }\end{array}$ & $\begin{array}{c}E_{i}(c) \\
(\mathrm{eV})\end{array}$ & $\begin{array}{c}E_{i}(\mu) \\
(\mathrm{eV})\end{array}$ & $\begin{array}{c}\Gamma_{i}(c) \\
(\mathrm{eV})\end{array}$ & $\begin{array}{c}\Gamma_{i}(\mu) \\
(\mathrm{eV})\end{array}$ & $\begin{array}{c}F_{i}(c) \\
(\mathrm{eV})\end{array}$ & $\begin{array}{c}F_{i}(\mu) \\
(\mathrm{eV})\end{array}$ \\
\hline$E_{1}$ & 2.92 & 2.92 & 0.19 & 0.53 & 2.22 & 2.56 \\
\hline$E_{1}+\Delta_{1}$ & $\begin{array}{l}3.12 \\
3.39 \\
3.86 \\
4.56\end{array}$ & $\begin{array}{l}3.16 \\
3.45 \\
3.94 \\
4.59\end{array}$ & $\begin{array}{l}0.34 \\
0.68 \\
0.92 \\
0.75\end{array}$ & $\begin{array}{l}0.50 \\
0.67 \\
0.98 \\
0.95\end{array}$ & $\begin{array}{l}3.16 \\
3.88 \\
4.09 \\
7.22\end{array}$ & $\begin{array}{l}2.82 \\
3.21 \\
3.65 \\
7.69\end{array}$ \\
\hline$E_{2}$ & $\begin{array}{l}4.85 \\
6.1\end{array}$ & $\begin{array}{l}4.87 \\
6.3\end{array}$ & $\begin{array}{l}0.39 \\
2.5\end{array}$ & $\begin{array}{l}0.78 \\
1.9\end{array}$ & $\begin{array}{c}4.82 \\
9.3 \\
\epsilon_{\infty}=1.55\end{array}$ & $\begin{array}{c}5.53 \\
10.2 \\
\epsilon_{\infty}=2.38 \\
\end{array}$ \\
\hline
\end{tabular}


cuss these.

By far, the most pronounced microcrystallinityinduced $(c \rightarrow \mu)$ changes in Table II are those for the $\Gamma$ values in the three labeled rows. The linewidth parameter nearly triples for $E_{1}$, increases by $50 \%$ for $E_{1}+\Delta_{1}$, and doubles for $E_{2}$. Among the oscillator parameters, these three $\Gamma_{i}$ 's experience the largest relative changes from the crystalline values. The finite-size effects, which cause the $\mu$-GaAs spectra of Figs. $4-6$ to depart from the crystal spectrum (the effect increasing with decreasing microcrystallite size, in the sequence $a \rightarrow b \rightarrow c \rightarrow d$ ), are concentrated in these three quantities. Thus the changes in these $\Gamma$ 's must be taken seriously, since they epitomize the $c \rightarrow \mu$ spectral evolution exhibited in Figs. 4-6.

Figure 7 displays a graphic representation of the microcrystallinity-induced variation in these linewidth parameters. The horizontal axis is $1 / L$, the reciprocal of the characteristic crystallite size. Evidence that $L^{-1}$ provides a useful measure of microcrystallinity was found in the earlier Raman-scattering studies on these materials. ${ }^{22}$ The three small- $L$ values plotted at the right and center of Fig. 7 correspond to the Raman-derived $L$ 's listed in Table I for the three high-fluence implants. The points plotted on the left-hand axis correspond, of course, to the bulk crystal $(c-G a A s, L=\infty)$. The $L$ value for the lowfluence implant (first row of Table I) was not independently obtained, but is an interpolated estimate based on the observed $\Gamma$ and the linear $\Gamma\left(L^{-1}\right)$ dependence for the case of the $E_{1}$ oscillator. Using the crude estimate so obtained for this $L$ yields the two points plotted for $E_{1}+\Delta_{1}$ and $E_{2}$, which are seen to be reasonably consistent with the rest of the data.

For each interband-transition oscillator, we may express the linewidth $\Gamma$ as the sum of two terms:

$$
\Gamma=\Gamma_{0}+\Gamma_{\text {size }} \text {. }
$$

Here, $\Gamma$ is the linewidth measured for a microcrystalline sample ( $\mu$-GaAs), $\Gamma_{0}$ is the linewidth measured for the bulk crystal ( $c$-GaAs), and $\Gamma_{\text {size }}$ is the additional sizeeffect broadening contributed to the linewidth in GaAs microcrystals by the effect of finite size. From Fig. 7 it is clear that, within the experimental error, our results show that $\Gamma_{\text {size }}$ is proportional to the reciprocal of the characteristic microcrystal size:

$$
\Gamma_{\text {size }}=A / L,
$$

so that

$$
\Gamma=\Gamma_{0}+A / L
$$

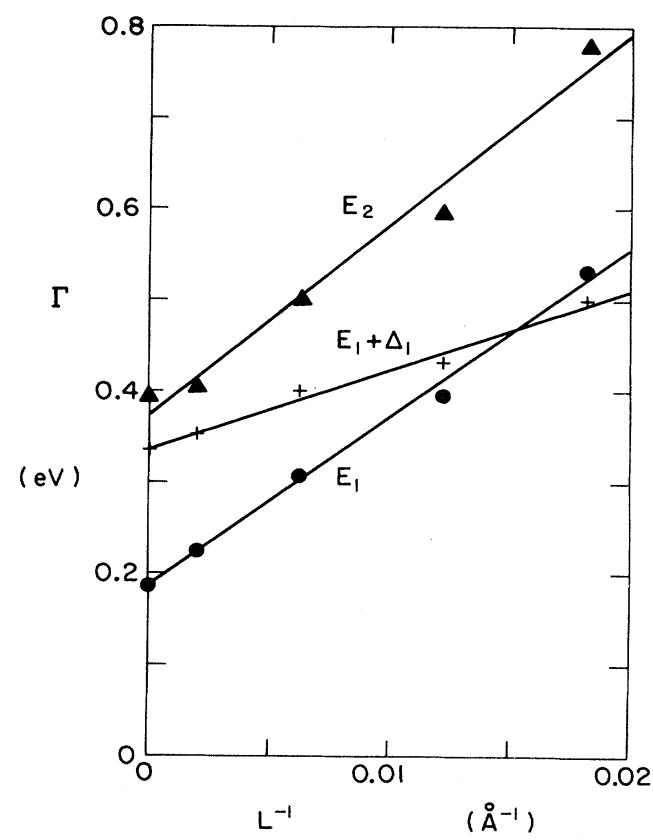

FIG. 7. Spectral linewidth as a function of the reciprocal of the characteristic microcrystallite size for the three prominent interband features in the $\mu$-GaAs spectra of Figs. 4-6. The straight lines are fits to the data.

Here, $A$ is a constant corresponding to the slope of the appropriate line in Fig. 7. We list the $A$ values in Table III.

Before discussing interpretations of and mechanisms for the second term on the right-hand side of Eq. (9), we should first mention the origin of the first term, the "intrinsic" (bulk crystal) linewidth $\Gamma_{0}$. Sharp features in the dielectric function originate from sharp features in the joint density of states for direct (or "vertical" or kconserving) electronic transitions from the valence band to the conduction band. The joint density of states is a continuous function $\rho_{\mathrm{cv}}(E)$ and the shape (and effective spectral width) of each critical-point feature arises from the band structure $E_{\mathrm{cv}}(\mathbf{k})$ in the vicinity of the contributing critical point(s) in $\mathbf{k}$ space. In addition, there are mechanisms in the bulk crystal (carrier-phonon interaction, carrier-carrier interactions, etc.) which serve to limit the lifetimes of the optically induced electron-hole excited states and which introduce additional broadening into

TABLE III. Excited-state lifetimes $\tau$ as limited by finite-size effects in $\mu$-GaAs, as well as carrier velocities $v$, estimated from the observed slopes $(A)$ of the linear relations connecting spectral linewidth $\Gamma$ and reciprocal microcrystallite size $L^{-1}$. The quantity $4 \pi(d E / d k)_{\max }$, estimated from the band structure of Ref. 24, is included for comparison to $A$.

\begin{tabular}{lcccc}
\hline $\begin{array}{l}\text { Interband } \\
\text { transition }\end{array}$ & $\begin{array}{c}\text { Expt. slope } A \\
{\left[d \Gamma / d L^{-1}(\mathrm{eV} \AA)\right]}\end{array}$ & $\begin{array}{c}4 \pi(d E / d k)_{\max } \\
(\mathrm{eV} \AA)\end{array}$ & $\begin{array}{c}v \\
(\mathrm{~cm} / \mathrm{s})\end{array}$ & $\begin{array}{c}\tau \text { (for } L=100 \AA) \\
(\mathrm{s})\end{array}$ \\
\hline$E_{1}$ & 18 & 16 & $2 \times 10^{7}$ & $2 \times 10^{-14}$ \\
$E_{1}+\Delta_{1}$ & 9 & 16 & $1 \times 10^{7}$ & $5 \times 10^{-14}$ \\
$E_{2}$ & 21 & 34 & $2 \times 10^{7}$ & $2 \times 10^{-14}$ \\
\hline \hline
\end{tabular}


the observed linewidth. The combined linewidth arising from $\rho_{\mathrm{cv}}(E)$ and the superimposed lifetime-limitation broadening is the "intrinsic" linewidth $\Gamma_{0}$ which characterizes the bulk-crystal interband feature in roomtemperature $c$-GaAs. In $\mu$-GaAs there is an additional broadening $\Gamma_{\text {size }}$ introduced by the microcrystalline graininess, as manifested in Fig. 7 and the second term on the right-hand side of Eqs. (7) and (9).

\section{MECHANISMS FOR THE FINITE-SIZE EFFECTS IN $\mu$-GaAs}

The discussion of the preceding section suggests that we seek a lifetime-limitation mechanism for the finite-size effects we see in $\mu$-GaAs. The proximity of the microcrystal boundary to the optically excited electron and hole provides a natural mechanism. We give a simple uncertainty-principle argument for the spectral effect of the carrier-boundary interaction.

The basic idea is that a collision with the microcrystal boundary scatters an optically excited carrier into another state, limiting the excited-state lifetime. Let the excited carriers possess a characteristic velocity $v$. Then a rough estimate of the average time $\tau$ it takes for an excited electron (or hole) to "feel" the boundary in a microcrystal of size $L$ is

$$
\tau=(L / 2) / v \text {. }
$$

If we invoke the uncertainty principle, $\tau \Delta E=h$, and identify $\Delta E$ with $\Gamma_{\text {size }}$, Eq. (7) becomes

$$
\Gamma=\Gamma_{0}+4 \pi \hbar v \frac{1}{L}
$$

This equation has precisely the form of the experimentally observed relation, Eq. (9). Equating $A$ with $4 \pi \hbar v$ yields

$$
v=\frac{A}{4 \pi \hbar}
$$

We may note that a relation similar to Eq. (11) has been used in connection with the optical properties of granular metals, in which case the linewidth term corresponds to the Drude collision rate and $v$ corresponds to the Fermi velocity. ${ }^{23}$

Table III lists the velocities implied by Eq. (12) and the experimental values for $A$. Also included in Table III are values of the size-effect lifetime $\tau(L)$ for $L=100 \AA$, representative of our range of samples. These short lifetimes, in the $10^{-14}$-s range, are estimated from Eq. (10) and the optically derived velocities [Eq. (12)]. In this regime of microcrystallinity, the finite-size effect is the dominant excited-state lifetime limitation.

A different view of the finite-size mechanism is obtained by considering the departure of the electron states from Bloch states. In a microcrystal of size $L$, each state acquires a $\mathbf{k}$-space spread $\Delta k$ of order $2 \pi / L$. This corresponds to the momentum spread $\hbar \Delta k$ mandated by the position-momentum uncertainty principle for an electron confined within $L$. The wave-vector spread $\Delta k$, in turn, gives rise to a spread in the energy of interband transitions, as shown in Fig. 8. (a)

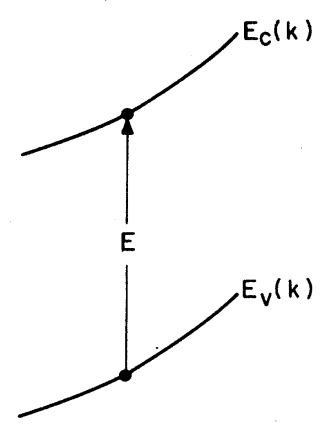

(b)

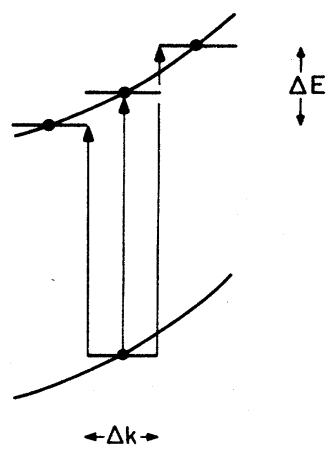

FIG. 8. Direct interband electronic transitions, in the vicinity of a critical point, in a bulk crystal, (a), and in a microcrystallite, (b). The k-vector broadening due to the finite crystallite size in (b) results in the broadening of the transition energy.

Figure $8(\mathrm{a})$ indicates a direct $\left(\mathbf{k}_{c}=\mathbf{k}_{v}\right)$ transition in $c$ GaAs in the vicinity of a critical point at which the bands are parallel. All vertical transitions near the critical point contribute optical-absorption processes at a welldefined energy. In $\mu$-GaAs, significant wave-vector spread $\Delta k$ is present and the effect is shown in Fig. 8(b). An energy spread $\Delta E$ arises in the transition via the energy-band dispersion $d E / d k$. Using $2|d E / d k| \Delta k$ for $\Delta E, 2 \pi / L$ for $\Delta k$, and identifying $\Delta E$ with the size-effect broadening $\Gamma_{\text {size }}$ of Eq. (7) yields

$$
\Gamma=\Gamma_{0}+4 \pi\left|\frac{d E}{d k}\right| \frac{1}{L} .
$$

Equation (13), like Eq. (11), is a linear relation between $\Gamma$ and $L^{-1}$, in agreement with experiment [Fig. 7, Eq. (9)]. The equivalence of Eqs. (13) and (11) can be seen by associating $v$ of Eq. (11) with the group velocity $\hbar^{-1} d E / d k$ corresponding to a wave packet constructed from states near $k$.

Equation (13) allows us to connect our results to the band structure of GaAs. The k-space gradient of $E(\mathbf{k})$, which determines the second term in Eq. (13), can be estimated from the bands in those regions of the Brillouin zone which dominate the contribution to each optical feature. The question of identifying the $\mathbf{k}$-space regions which give rise to the $E_{1}, E_{1}+\Delta_{1}$, and $E_{2}$ features has long been studied, ${ }^{13,24-27}$ and yet is incompletely resolved. We make the following consensus-type assumptions, and rely on the energy band structure of Cohen and Chelikowsky $^{24}$ for extracting numerical estimates. We assume that the $E_{1}$ and $E_{1}+\Delta_{1}$ transitions occur along the $\Lambda$ directions, including the $L$ points, and that the $E_{2}$ transitions occur near the $X$ and $K$ points and along the $\Sigma$ directions. ${ }^{28}$ Under these assumptions, we can estimate a range of values for $|d E / d k|$ for each transition. The minimum value is zero in each case, corresponding to the horizontal bands at the symmetry points ${ }^{25} L$ (for $E_{1}$ and $E_{1}+\Delta_{1}$ ) and $X$ (for $E_{2}$ ). The maximum gradient for $E_{1}$ 
and $E_{1}+\Delta_{1}$ occurs along $\Lambda$, about halfway between $\Gamma$ and $L$, and for $E_{2}$ it occurs near $K$. These maximum values are included in Table III in the form $4 \pi|d E / d k|$, in order to provide a comparison to our experimental $A$ values [equating the $L^{-1}$ coefficients in Eqs. (9) and (13)]. Considering the approximations contained in our model, the rough agreement is reasonable and supports our overall picture.

A detail worthy of comment is the experimental difference we find between the behaviors of the size-effect broadenings of the $E_{1}$ and $E_{1}+\Delta_{1}$ peaks. Since this doublet arises from the spin-orbit splitting of the valence band, it is generally presumed that both peaks arise from transitions in the same region of $\mathbf{k}$ space (hence the equal entries in the middle column of Table III). However, the difference that we observe in the finite-size effects on $E_{1}$ and $E_{1}+\Delta_{1}$ suggests that the contributing $\mathbf{k}$-space regions are shifted relative to each other. It is noteworthy that a recent detailed study of the effect of temperature on the interband spectra of $c$-GaAs finds that the $E_{1}+\Delta_{1}$ linewidth increases more slowly with increasing temperature than does the $E_{1}$ linewidth, ${ }^{29}$ a result which is in the same direction as our finding for the effect on linewidth of finite microcrystal size.

\section{SUMMARY}

Finite-size effects on electronic excitations have been experimentally identified in the interband spectra of microcrystalline GaAs. Visible-ultraviolet reflectivity measurements were carried out on a series of unannealed $\mathrm{Be}^{+}$-implanted GaAs samples in which the implantation-induced damage layer is known (from previous Raman work) to consist of a fine-grain mixture of amorphous and microcrystalline GaAs. Oscillator analysis was used to derive the optical dielectric function of the implanted layer from the observed reflectivity spectrum. Then, by inverting the effective-medium approximation, we have extracted (from the optical properties of the two-phase damage layer) the optical functions of the microcrystalline component, $\mu$-GaAs. The optical properties of $\mu$-GaAs are seen (Figs. 4-6) to differ appreciably from those of the bulk crystal (c-GaAs), with the difference increasing as the characteristic microcrystallite size $(L)$ decreases.
The appropriate quantitative characterization of the evolution of the optical properties of $\mu$-GaAs with decreasing $L$ is given in terms of the spectral linewidths of the features associated with the $E_{1}, E_{1}+\Delta_{1}$, and $E_{2}$ direct interband transitions. As discussed in Sec. V, the spectral changes induced by small $L$ are found to be concentrated in these three linewidths. These linewidths increase linearly and rapidly (Fig. 7) with $L^{-1}$, the inverse microcrystal size. When $L=100 \AA$ the size-effect increase in linewidth is about $0.2 \mathrm{eV}$ for the $E_{1}$ and $E_{2}$ peaks, comparable to the intrinsic bulk-crystal roomtemperature linewidth.

A simple theory based on the uncertainty principle is found to semiquantitatively account for our observations on finite-size effects in $\mu$-GaAs, notably the linear dependence of linewidth on $L^{-1}$ as well as the magnitude of this dependence. Small microcrystal size implies a short time for excited carries to reach, and to be scattered by, the microcrystal boundary. This limits the excited-state lifetime, and thus broadens the excited-state energy. Our results indicate (Table III) a severe lifetime limitation, of order $3 \times 10^{-14} \mathrm{~s}$, for microcrystallinity in the $100-\AA$ range. The velocities indicated by the experimentally derived lifetimes are consistent with band-structure group velocities in GaAs. An alternative argument, also based on the uncertainty principle, can be expressed in terms of $\mathbf{k}$-space broadening of electron states: a $\mathbf{k}$-space spread of order $2 \pi / L$ is implied by microcrystal size $L$. This viewpoint yields, via $E(\mathbf{k})$ energy-band dispersion, an equivalent linear relation between linewidth and $L^{-1}$, in agreement with experiment.

\section{ACKNOWLEDGMENTS}

The authors are indebted to A. Purdes, S. Matteson, and T. Brandon of the Texas Instruments Materials Science Laboratory for the $\mathrm{Be}^{+}$-implanted GaAs samples, to D. E. Aspnes for providing us with his unpublished dielectric-function data on $a$-GaAs made amorphous by implantation, to Y. Y. Wang, M. Holtz, J. G. Dillard, and L. C. Burton for valuable discussions, and to D. M. Hoffman for invaluable advice on spectroscopic instrumentation. This work was supported in part by Texas Instruments and the Virginia Center for Innovative Technology.
${ }^{1}$ M. Erman, J. B. Theeten, P. Chambon, S. M. Kelso, and D. E. Aspnes, J. Appl. Phys. 56, 2664 (1984).

${ }^{2}$ M. Holtz, R. Zallen, O. Brafman and S. Matteson, Phys. Rev. B 37, 4609 (1988).

${ }^{3}$ S. Pang, J. Electrochem. Soc. 133, 784 (1986).

${ }^{4}$ G. F. Feng, M. Holtz, R. Zallen, J. M. Epp, J. G. Dillard, E. Cole, P. Johnson, S. Sen, and L. C. Burton, in Materials Modification and Growth Using Ion Beams, Vol. 93 of Materials Research Society Symposium Proceedings, edited by U. J. Gibson, A. E. White, and P. P. Pronko (MRS, Pittsburgh, 1987), p. 381.

${ }^{5}$ M. Gargouri, B. Prevot, and C. Schwab, J. Appl. Phys. 62,
3902 (1987).

${ }^{6}$ K. S. Jones, D. K. Sadana, S. Prussin, J. Washburn, E. R. Weber, and W. J. Hamilton, J. Appl. Phys. 63, 1414 (1988).

${ }^{7}$ L. L. Liou, W. G. Spitzer, J. E. Fredrickson, and S. Kwun, J. Appl. Phys. 59, 1927 (1986).

${ }^{8}$ S. Prussin, D. I. Margolese, and R. Tauber, J. Appl. Phys. 57, 180 (1985).

${ }^{9}$ S. Kumar, B. Drevillon, and C. Godet, J. Appl. Phys. 60, 1542 (1986).

${ }^{10}$ H. Richter and L. Ley, J. Appl. Phys. 52, 7281 (1981); Z. Iqbal and S. Veprek, J. Phys. C 15; 377 (1982); H. N. Liu, D. Pfost, and J. Tauc, Solid State Commun. 50, 987 (1984). 
${ }^{11}$ S. Veprek, F. A. Sarott, and Z. Iqbal, Phys. Rev. B 36, 3344 (1987); L. Chen, J. Tauc, and Z. Vardeny, ibid. 39, 5121 (1989).

${ }^{12}$ D. E. Aspnes and A. A. Studna, Phys. Rev. B 27, 985 (1983).

${ }^{13}$ P. Lautenschlager, M. Garriga, S. Logothetidis, and M. Cardona, Phys. Rev. B 35, 9174 (1987).

${ }^{14}$ D. E. Aspnes (private communication); also see D. E. Aspnes, S. M. Kelso, C. G. Olson, and D. W. Lynch, Phys. Rev. Lett. 48, 1863 (1982).

${ }^{15}$ Our own experiments on sputtered and evaporated $a$-GaAs thin films reveal significant variation of the overall magnitude of the reflectivity with method of sample preparation, but in each case the $a-\mathrm{GaAs}$ spectrum is bland and featureless like that shown in Fig. 1. Since the optical quality of the implantation-amorphized GaAs of Ref. 14 was high, like that of our implanted samples, those data provide a suitable $a$ GaAs spectrum for the comparison.

${ }^{16}$ W. G. Spitzer and D. A. Kleinman, Phys. Rev. 121, 1324 (1961).

${ }^{17}$ H. W. Verleur, J. Opt. Soc. Am. 58, 1356 (1968).

${ }^{18}$ Math/PC-Library (User's Manual), IMSL, 1984.

${ }^{19}$ H. Richter, Z. P. Wang, and L. Ley, Solid State Commun. 39,
625 (1981).

${ }^{20}$ D. E. Aspnes, Thin Solid Films 89, 249 (1982).

${ }^{21}$ A. G. Bruggeman, Ann. Phys. (Leipzig) 24, 636 (1935).

${ }^{22}$ See Fig. 8 of Ref. 2.

${ }^{23}$ S. Norrman, T. Andersson, C. G. Granqvist, and O. Hunderi, Phys. Rev. B 18, 674 (1978). See Eq. (26) on p. 683.

${ }^{24}$ M. L. Cohen and J. R. Chelikowsky, in Handbook on Semiconductors 1, edited by W. Paul (North-Holland, Amsterdam, 1982); J. R. Chelikowsky and M. L. Cohen, Phys. Rev. B 14, 556 (1976).

${ }^{25}$ See, for example, J. C. Phillips, in Solid State Physics, edited by F. Seitz and D. Turnbull (Academic, New York, 1966), Vol. 18, p. 55.

${ }^{26}$ H. Ehrenreich, H. R. Philipp, and J. C. Phillips, Phys. Rev. Lett. 8, 59 (1962).

${ }^{27}$ D. L. Greenaway, Phys. Rev. Lett. 9,97 (1962).

${ }^{28}$ The notation used here for points and lines in the Brillouin zone is the standard one. See, for example, R. Zallen, in Handbook on Semiconductors Vol. 1, edited by W. Paul (North-Holland, Amsterdam, 1982).

${ }^{29}$ See the next-to-last column of Table II of Ref. 13. 